\title{
Protons Spectrum from the MAGIC Telescopes data
}

\section{P. Temnikov ${ }^{a,}{ }^{*}$, V. Verguilov ${ }^{a}$, G. Maneva ${ }^{a}$, R. Mirzoyan ${ }^{b}$, D. Baack ${ }^{c}$ on behalf of the MAGIC Collaboration ${ }^{\dagger}$}

${ }^{a}$ Institute for Nuclear Research and Nuclear Energy, Bulgarian Academy of Sciencies, 1084, Sofia, Bulgaria

${ }^{b}$ Max-Planck-Institute for Physics (Werner-Heisenberg-Institute),

Föhringer Ring 6, D-80805, Munich, Germany

${ }^{c}$ Fakultät Physik, Technische Universität Dortmund,

D-44227, Dortmund, Germany

E-mail: petar.temnikov@gmail.com, vassil.verguilv@gmail.com, maneva@inrne.bas.bg, razmik.mirzoyan@mpp.mpg.de, dominik.baack@tu-dortmund.de

Imaging Atmospheric Cherenkov telescopes (IACTs) are designed to detect cosmic gamma rays. As a by-product, IACTs detect Cherenkov flashes generated by millions of hadronic air showers every night. We present the proton energy spectrum from several hundred $\mathrm{GeV}$ to several hundred $\mathrm{TeV}$, retrieved from the hadron induced showers detected by the MAGIC telescopes. The protons are discriminated from $\mathrm{He}$ and other heavy nuclei by means of using machine learning classification. The energy estimation is based on a specially developed deep neural network regressor. In the last decade, Deep Learning methods gained much interest in the scientific community for their ability to extract complex relations in data and process large datasets in a short time. The proton energy spectrum obtained in this work is compared to the spectra obtained by dedicated cosmic ray experiments.

ICRC 2021, 37th International Cosmic Ray Conference

12-23 July 2021

Online-Berlin, Germany

"Speaker

ta complete list of the MAGIC Collaboration authors can be found at the end of the proceedings 


\section{Introduction}

The recent results in cosmic ray physics demonstrate that we are entering an era of precision experiments. The experiments CREAM [1], NUCLEON [2] and CALET [3] found evidence for some structure above $10 \mathrm{TeV}$ in the proton spectra. The DAMPE experiment with $2 \frac{1}{2}$ years of selected measurements published precise spectra of cosmic nuclei that show not trivial structure [4].

Over the last two decades, imaging air Cherenkov telescopes (IACT) have collected huge amounts of air shower data, most of which are generated by charged nuclei. It is generally believed that data from IACTs, which are designed to preferentially detect gamma induced showers, are not well-suited for studying charged cosmic-ray particles. However, there have been few attempts to extract the proton spectrum from such data. For example, the experiment HEGRA successfully extracted the proton spectrum, but in a narrow range of energies [5]. Later independent researches of the proton spectrum were conducted on the basis of H.E.S.S. measurements [6,7]. Measurements of the cosmic iron spectrum were performed by the experiments H.E.S.S. and VERITAS [9,10,11], for events selection using the direct Cherenkov radiation of the heavy nuclei before the start of induced atmospheric showers; these based on the method proposed by Kieda et al. [8].

The purpose of this study is to show that the background data detected by the two MAGIC telescopes, which are generated in the atmosphere mainly by charged cosmic particles, can be successfully used to study the spectra of these nuclei in a wide range of energies. The method presented here needs no assumption about the estimated spectrum and thus allowed us to perform a detailed search of the spectral features. The proton spectrum obtained in the region $700 \mathrm{GeV}-500 \mathrm{TeV}$ is well-compatible with the precision of DAMPE and CREAM-III proton spectrum measurements.

\section{Data and Simulations Sets}

For this study we have used about 70 hours of observational data taken in 2016 and 2017. The Monte Carlo (MC) simulations were done for the 3 most abundant components of charged cosmic rays: protons, helium and iron. At energy $10 \mathrm{TeV}$ their ratio is about 1:1:0.2. In future we plan to use Monte Carlo simulation of $\mathrm{C}$ and $\mathrm{O}$ nuclei.

The showers were simulated within the impact parameter range of up to $1500 \mathrm{~m}$ and the viewing angle of 4 degrees in the following energy ranges and statistics in the Corsika code (version 6.990) :

- Protons: $70 \mathrm{GeV}-500 \mathrm{TeV}$ - about $2.5 \cdot 10^{8}$ events.

- He: $140 \mathrm{GeV}-2000 \mathrm{TeV}$ - about $1.2 \cdot 10^{8}$ events

- Fe: $400 \mathrm{GeV}-6000 \mathrm{TeV}$ - about $5 \cdot 10^{7}$ events

For the analysis we selected only events that have passed trigger conditions in the energy range $700 \mathrm{GeV}-5000 \mathrm{TeV}$. Finally, about 80000,56000 and 28000 simulated events for proton, helium and iron correspondingly survived for the analysis. The used real data after data quality cuts includes about 9.6 million events collected during 70 hours of observations in 2016 and 2017. 


\section{Analysis method}

We use Supervised Feed-forward Neural Networks with Back Propagation method for error minimization to create the energy regressor and event classifier. As input variables we used Hillas parameters and additional variables, traditional for the MAGIC experiment. We don't use the convolution layers method, because it is too sensitive to small differences between simulation and real data.

The network architecture applied for the energy reconstruction consists of 1 input layer of 21 nodes, 3 hidden layers of 16, 8, 4 hidden nodes and 1 output layer of 1 node. Although corresponding networks were created, trained and validated for any of the three mentioned above types of nuclei, in the proton flux determination for He and Fe we used energy obtained with the network, trained to reconstruct the proton energy.

For the separation of the protons from all the other nuclei we created two completely different networks, aiming at the discrimination of the proton induced shower against helium and against iron induced showers. Both networks have the same architecture consisting of 1 input, 4 hidden and 1 output layers with 36, 28, 18, 10, 5, 1 nodes correspondingly. These were trained independently on one half of the corresponding MC events and validated on the other half of the $\mathrm{MC}$ events, not used in the training procedure. These two networks were applied to all the real data samples.

\subsection{Energy reconstruction}

The results obtained for the MC protons from the described above energy regressor are shown in the figures below. The energy resolution is estimated by plotting $\left(\mathrm{E}_{\text {true }}-\mathrm{E}_{\text {estimated }}\right) / \mathrm{E}_{\text {true }}$ in bins of $\mathrm{E}_{\text {true }}$ and fitting this distribution by a Gaussian function.

On the below figure the top left panel presents the simulated and reconstructed spectra. On the top right panel the energy resolution, integrated for all energies is presented. The bottom left and right panels are for energy resolution and energy bias as functions of the simulated energy.
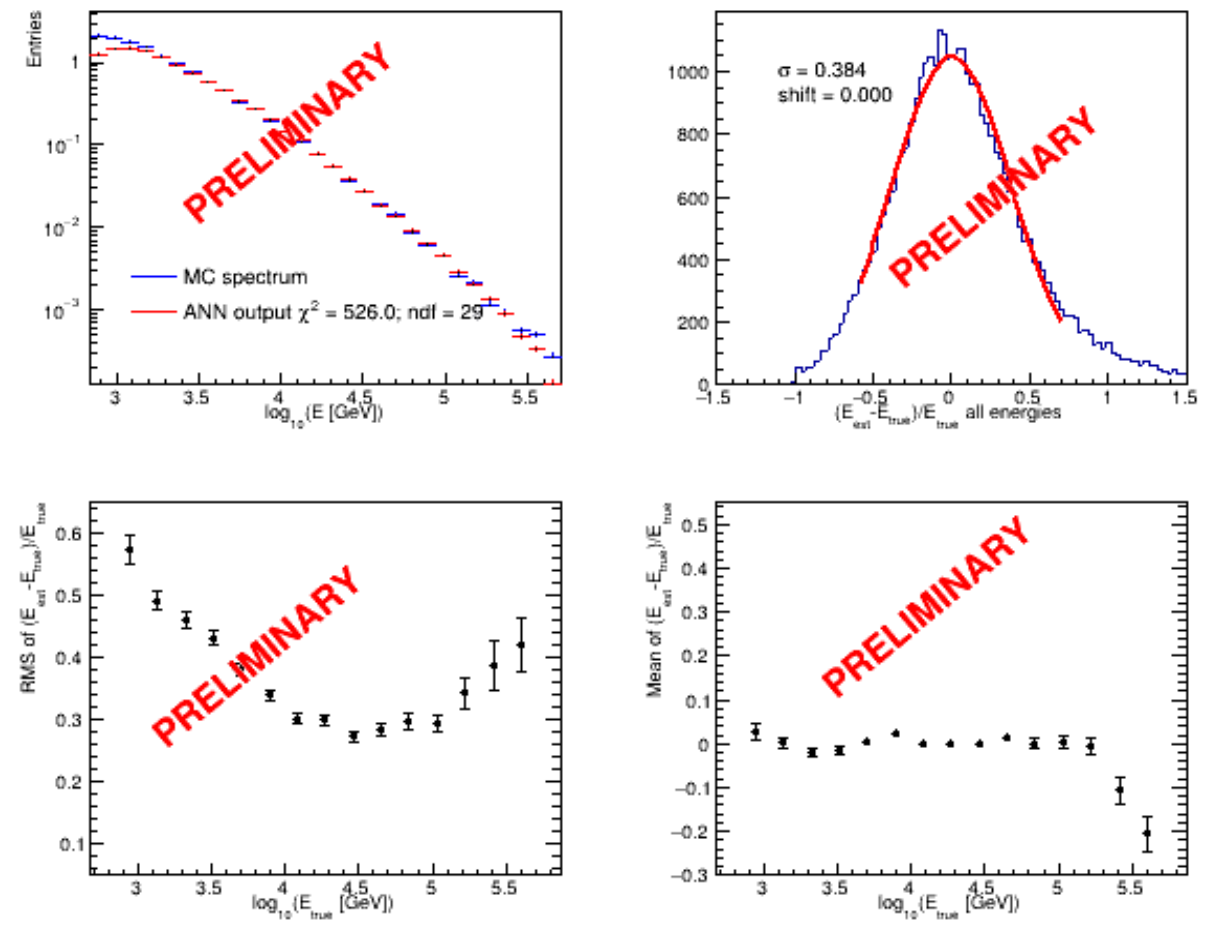

Figure 1: Energy estimation results 
On the next figure the migration matrix between true and estimated energies is presented. It is normalized to 1 in every row of true energy, including overflow and underflow bins. Only bins with contents $>0.003$ are plotted.

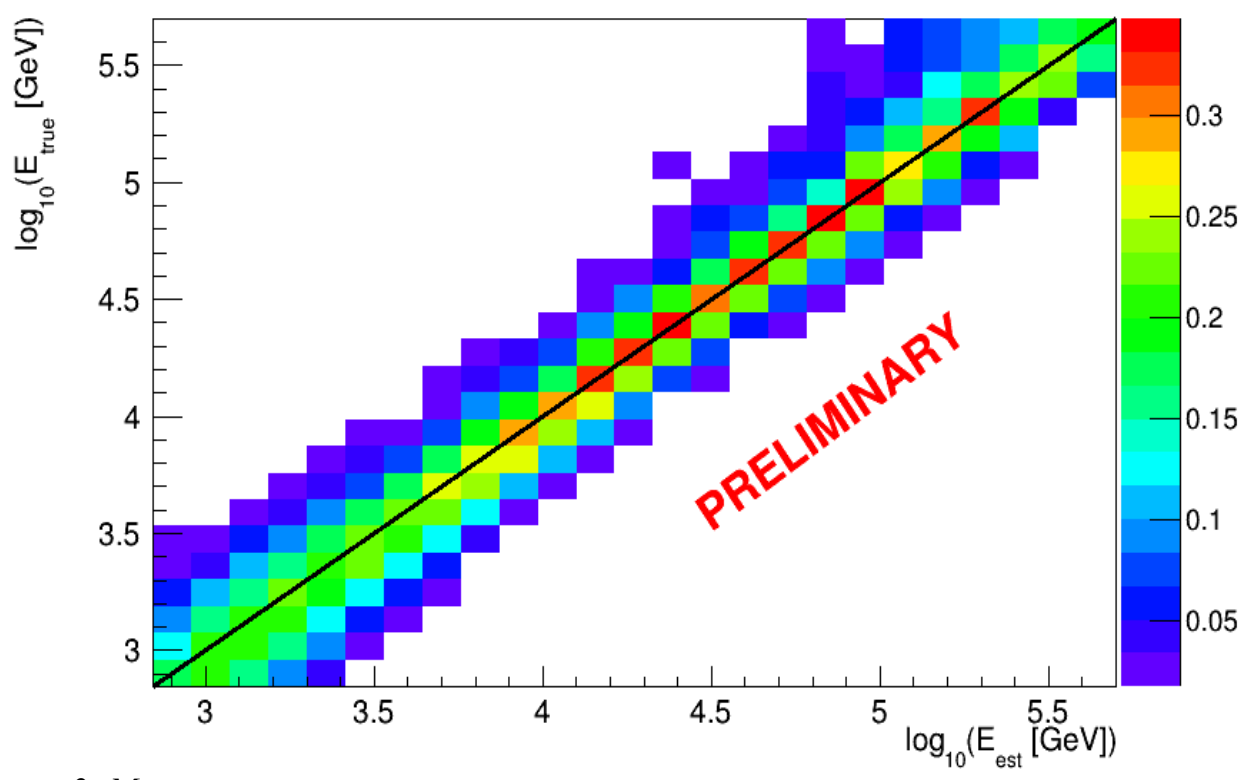

Figure 2: Migration matrix

\subsection{Classification}

On the figures below the outputs of 2 classification networks ( $\mathrm{p}-\mathrm{He}$ and $\mathrm{p}-\mathrm{Fe}$ ) for 3 elements ( $\mathrm{p}, \mathrm{He}$ and $\mathrm{Fe}$ ) are shown. For the p-Fe classifier we present the graphs also in logarithmic scale for better visibility.
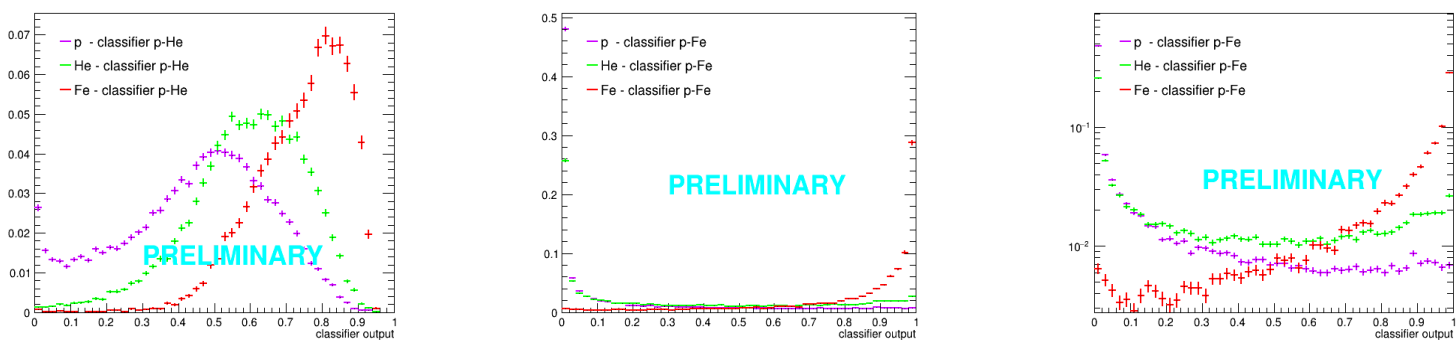

Figure 3: Classification results

\subsection{Flux calculation}

In the case of 3 components (proton, $\mathrm{He}, \mathrm{Fe}$ ) and two neural networks for classification ( $\mathrm{p}-\mathrm{He}$ and $\mathrm{p}-\mathrm{Fe}$ ) the number of protons $\mathrm{N}_{\text {protons }}$ may be estimated as:

$$
N_{\text {protons }}=\frac{\varepsilon_{1} \cdot\left(p_{2, \mathrm{He}}-p_{2, \mathrm{Fe}}\right)-\varepsilon_{2} \cdot\left(p_{1, \mathrm{He}}-p_{1, \mathrm{Fe}}\right)+p_{1, \mathrm{He}} \cdot p_{2, \mathrm{Fe}}-p_{2, \mathrm{He}} \cdot p_{1, \mathrm{Fe}}}{p_{1, \mathrm{p}} \cdot\left(p_{2, \mathrm{He}}-p_{2, \mathrm{Fe}}\right)-p_{2, \mathrm{p}} \cdot\left(p_{1, \mathrm{He}}-p_{1, \mathrm{Fe}}\right)+p_{1, \mathrm{He}} \cdot p_{2, \mathrm{Fe}}-p_{2, \mathrm{He}} \cdot p_{1, \mathrm{Fe}}} \frac{N}{E f f}
$$


Here $\mathbf{p}_{1, i}$ and $\mathbf{p}_{2, \mathrm{i}} \mathrm{i}=\mathrm{p}, \mathrm{He}, \mathrm{Fe}$ are the probabilities to classify a shower as proton-like in the first and second binary classifiers for the three MC samples, $\boldsymbol{\varepsilon}_{1}$ and $\boldsymbol{\varepsilon}_{2}$ are the fractions of proton-like events selected from $\mathbf{N}$ real events by the first and second classifiers. The efficiency:

$$
\text { Eff }(E, \cos (\theta), \text { Zenith })=N_{\text {selected }} / N_{\text {simulated }}
$$

is a detection probability, calculated for $\mathrm{MC}$ proton showers, where $\mathrm{N}_{\text {selected }}$ represents the number of events that passed the trigger and analysis cuts, and $\mathrm{N}_{\text {simulated }}$ is the number of simulated showers.

All quantities in this formula are functions of energy, cosine of proton's arriving angle (angle between proton and telescope axis) and zenith angle of telescope pointing.

Statistical errors for probabilities $\mathrm{p}$ and fractions $\varepsilon$ are calculated from the corresponding binomial distributions. The statistical error of $\mathbf{N}_{\text {protons }}$ is estimated using the approximate error propagation formula keeping only the first two terms of the Taylor series. Due to the limited statistics of MC (specially for $\mathrm{Fe}$ ) the main contributions to the total error are due to uncertainties of quantities $\mathbf{p}_{\mathbf{i}, \mathbf{j}}$ (determined from simulated events). There exist statistical correlations between random variables corresponding to events of one type classified in both networks, i.e. for $\mathbf{p}_{\mathbf{1}, \mathrm{i}}$ and $\mathbf{p}_{\mathbf{2}, \mathbf{i}}$ for equal $\mathrm{i}=\mathrm{p}, \mathrm{He}, \mathrm{Fe}$ and between $\boldsymbol{\varepsilon}_{\mathbf{1}}$ and $\boldsymbol{\varepsilon}_{\mathbf{2}}$. These correlations were taken into account by calculating the final value of the statistical error of the estimated number of protons $\mathbf{N}_{\text {protons. }}$

The flux per unit energy/surface/time/angle is calculated as

$$
F(E, \cos (\theta), \text { Zenith })=N_{\text {protons }} /\left(\pi \cdot I^{2} \cdot T \cdot d E \cdot 2 \cdot \pi \cdot(1-\cos (V))\right)
$$

where $\mathrm{T}$ is the observation time, $\mathrm{I}$ is the radius of the simulated area, $\mathrm{V}$ is the maximum simulated angle and $\Delta \mathrm{E}$ is the energy bin width.

\section{Results}

\subsection{Energy spectrum}

The energy spectrum was estimated as it was described in the previous paragraph. It is important to perform an unfolding of the energy distribution, especially because the energy resolution in our case is about $30 \%$. We used the TUnfold software [12], which is included in the ROOT package. The method is based on the least-square fitting and the Tikhonov regularization method. The preliminary measured and unfolded spectra are presented in the slides for the talk at this conference. The value of the measured flux changes by 7 orders of magnitude and is in good agreement with the fluxes published by the experiments CREAM [1] and DAMPE [4].

In order to demonstrate the stability of our results, we divided the data set into two subsamples measured in different years: 60 hours of observations in 2016 and 10 hours in 2017. The two spectra coincide with each other taking into account the statistical errors. The statistical uncertainties are comparable for both data sets which have rather different sizes. It demonstrates that our statistical uncertainty is dominated by the statistics of the MC simulations. 


\subsubsection{Flux vs Arriving angle}

Cosmic rays arrive uniformly in the considered energy range. Below the angular distributions are presented. In the left panel the distribution for the whole data set is shown, in the central and right panels the distributions for 2016 and 2017 are plotted.
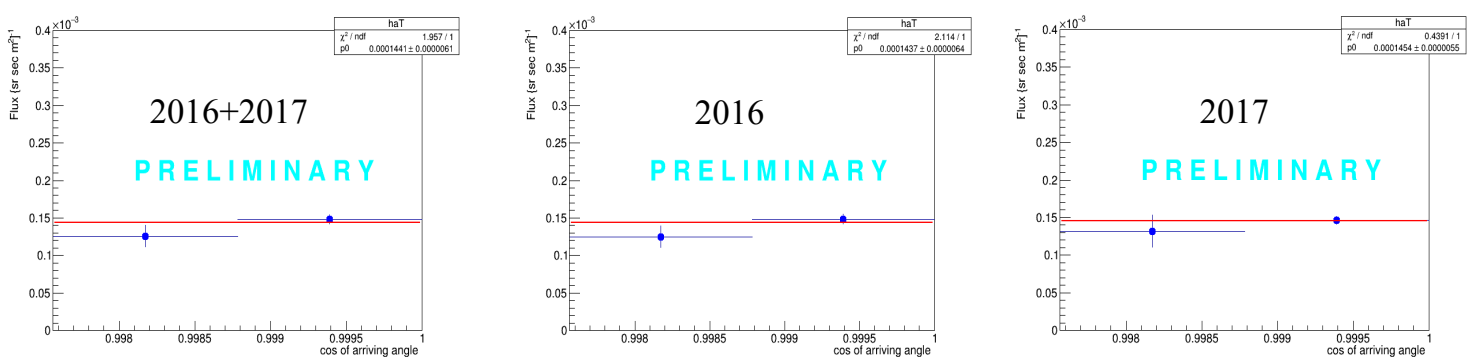

Figure 4: Arriving angle distributions

It is seen that the angular distribution is uniform and there is no significant difference between the estimated fluxes for the two different periods of observations.

\section{Consistency check}

If the flux calculation and the detection efficiency corrections are done properly, the flux values must be independent from the pointing telescope direction. Indeed, in our case the integrated flux for all the data and for the sub-samples from 2016 and 2017 are constant as a function of the zenith angle and are in good concordance for different cases: $1.345 \pm 0.008 \cdot 10^{-3}$, $1.356 \pm 0.008 \cdot 10^{-3}$ and $1.365 \pm 0.008 \cdot 10^{-3}$.
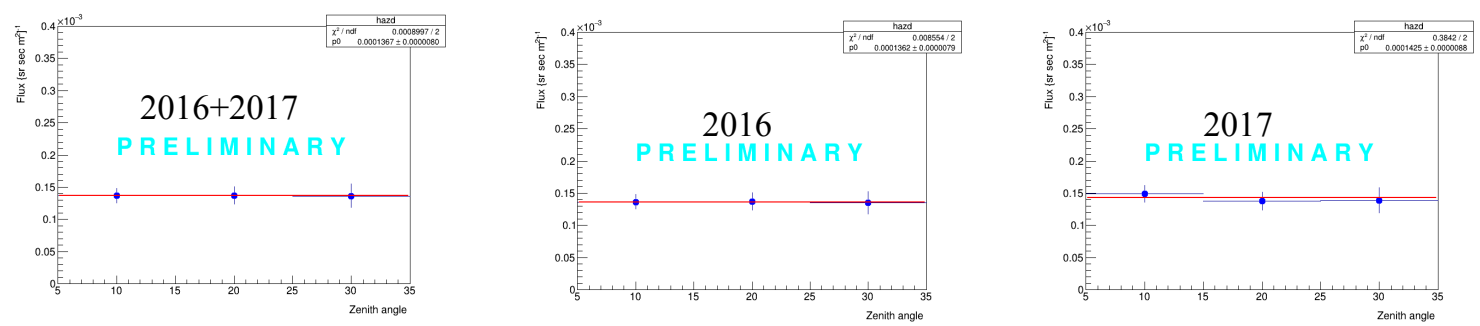

Figure 5: Zenith angle distributions

\section{Systematic uncertainties}

There are 3 main sources of systematic uncertainties:

- One is the influence of $\mathrm{C}$ and $\mathrm{O}$ nuclei which are not yet taken into account. Their contribution could be less than $10 \%$ to the proton flux

- Inaccuracy of hadronic model used in Corsika simulation code. According to R. D. Parsons and H. Schoorlemmer [13] it is estimated to be less than $10 \%$ in energy range $1-100 \mathrm{TeV}$

- Diverse uncertainties of the detector. From the typical MAGIC systematic effects [14] and from the flatness of the zenith and the arriving angles we estimate these effects as $\leq$ $30 \%$.

When we add these quadratically, the total systematic error turns out to be about $33 \%$. 


\section{Conclusions}

We demonstrated the practical feasibility of measuring the proton spectrum with high statistical accuracy in the energy range 1-500 $\mathrm{TeV}$ using a small fraction of data collected by the MAGIC telescopes system. The analysis method produces stable results. The next step could be the application of this method to the study of the spectra of different cosmic nuclei. Such study will permit high accuracy measurements of the energy spectra and elemental composition of cosmic nuclei by using the background data of IACT experiments.

\section{Acknowledgements}

We acknowledge the support of the agencies and organizations listed at: https://magic.mpp.mpg.de/acknowledgments_ICRC2021

\section{References}

1 Y. S. Yoon, et al. (CREAM Collaboration), Astrophys. J. 728, 122 (2011), arXiv:1102.2575 [astroph.HE]

2 E. Atkin, et al. (NUCLEON Collaboration), Sov. Phys. JETP Lett. 108, 5 (2018), arXiv:1805.07119 [astro-ph.HE]

3 O. Adriani, et al. (CALET Collaboration), Phys. Rev. Lett. 122, 181102 (2019), arXiv:1905.04229 [astro-ph.HE]

4 Q. An, et al. (DAMPE Collaboration), Science Advances 5, eaax3793(2019);

5 F. A. Aharonian et al. (HEGRA Collaboration), Phys. Rev. D59, 092003 (1999)

6 A. Schönwald, Diplomarbeit, Humboldt-Universität zu Berlin (2008)

7 D. Jankowsky, Thesis: PhD Erlangen - Nuremberg U. (2020)

8 D. B. Kieda, S. P. Swordy, and S. P. Wakely, Astropart. Phys. 15, 287 (2001). arXiv:astro-ph/0010554

9 F. A. Aharonian et al. (H.E.S.S. Collaboration), Phys.Rev. D75, 042004 (2007), arXiv:astro-ph/0701766, [ASTRO-PH].

10 S. Wissel, Observations of Direct Cerenkov Light in Ground-based Telescopes and the Flux of Iron Nuclei at TeV Energies, Ph.D. thesis, University of Chicago (2010)

11 A. Archer et al. (VERITAS Collaboration), Phys. Rev. D 98, 022009 (2018), arXiv:1807.08010 [astro-ph.HE]

12 S. Schmitt, JINST 7 (2012) T10003 [arXiv:1205.6201]

13 R. D. Parsons and H. Schoorlemmer, "Systematic differences due to high energy hadronic interaction models in air shower simulations in the $100 \mathrm{GeV}-100 \mathrm{TeV}$ range", Phys. Rev. D 100, 023010

14 Aleksic, J., Ansoldi, S., Antonelli, L. A., et al. Astropart. Phys., 72, 76 (2016) 


\section{Full Authors List: MAGIC Collaboration}

V. A. Acciari ${ }^{1}$, S. Ansoldi ${ }^{2,41}$, L. A. Antonelli ${ }^{3}$, A. Arbet Engels ${ }^{4}$, M. Artero ${ }^{5}$, K. Asano ${ }^{6}$, D. Baack ${ }^{7}$, A. Babic ${ }^{8}$, A. Baquero ${ }^{9}$, U. Barres de Almeida ${ }^{10}$, J. A. Barrio ${ }^{9}$, I. Batković ${ }^{11}$, J. Becerra González ${ }^{1}$, W. Bednarek ${ }^{12}$, L. Bellizzi ${ }^{13}$, E. Bernardini ${ }^{14}$, M. Bernardos ${ }^{11}$, A. Berti ${ }^{15}$, J. Besenrieder ${ }^{15}$, W. Bhattacharyya ${ }^{14}$, C. Bigongiari ${ }^{3}$, A. Biland ${ }^{4}$, O. Blanch ${ }^{5}$, H. Bökenkamp ${ }^{7}$, G. Bonnoli ${ }^{16}$, Ž. Bošnjak ${ }^{8}$, G. Busetto11, R. Carosi ${ }^{17}$, G. Ceribella ${ }^{15}$, M. Cerruti ${ }^{18}$, Y. Chai ${ }^{15}$, A. Chilingarian ${ }^{19}$, S. Cikota ${ }^{8}$, S. M. Colak ${ }^{5}$, E. Colombo ${ }^{1}$, J. L. Contreras ${ }^{9}$, J. Cortina ${ }^{20}$, S. Covino ${ }^{3}$, G. D’Amico ${ }^{15,42}$, V. D’Elia ${ }^{3}$, P. Da Vela $^{17,43}$, F. Dazzi ${ }^{3}$, A. De Angelis ${ }^{11}$, B. De Lotto ${ }^{2}$, M. Delfino ${ }^{5,44}$, J. Delgado ${ }^{5,44}$, C. Delgado Mendez ${ }^{20}$, D. Depaoli ${ }^{21}$, F. Di Pierro ${ }^{21}$, L. Di Venere ${ }^{22}$, E. Do Souto Espiñeira ${ }^{5}$, D. Dominis Prester ${ }^{23}$, A. Donini ${ }^{2}$, D. Dorner ${ }^{24}$, M. Doro ${ }^{11}$, D. Elsaesser ${ }^{7}$, V. Fallah Ramazani ${ }^{25,45}$, A. Fattorini ${ }^{7}$, M. V. Fonseca ${ }^{9}$, L. Font ${ }^{26}$, C. Fruck ${ }^{15}$, S. Fukami ${ }^{6}$, Y. Fukazawa ${ }^{27}$, R. J. García López ${ }^{1}$, M. Garczarczyk ${ }^{14}$, S. Gasparyan ${ }^{28}$, M. Gaug $^{26}$, N. Giglietto ${ }^{22}$, F. Giordano ${ }^{22}$, P. Gliwny ${ }^{12}$, N. Godinović ${ }^{29}$, J. G. Green ${ }^{3}$, D. Green ${ }^{15}$, D. Hadasch ${ }^{6}$, A. Hahn ${ }^{15}$, L. Heckmann ${ }^{15}$, J. Herrera ${ }^{1}$, J. Hoang ${ }^{9,46}$, D. Hrupec ${ }^{30}$, M. Hütten ${ }^{15}$, T. Inada ${ }^{6}$, K. Ishio ${ }^{12}$, Y. Iwamura ${ }^{6}$, I. Jiménez Martínez ${ }^{20}$ ，J. Jormanainen ${ }^{25}$, L. Jouvin ${ }^{5}$, M. Karjalainen ${ }^{1}$, D. Kerszberg ${ }^{5}$, Y. Kobayashi ${ }^{6}$, H. Kubo ${ }^{31}$, J. Kushida ${ }^{32}$, A. Lamastra ${ }^{3}$, D. Lelas ${ }^{29}$, F. Leone ${ }^{3}$, E. Lindfors ${ }^{25}$, L. Linhoff', $\mathrm{S}_{\text {. Lombardi }}^{3}$, F. Longo ${ }^{2,47}$, R. López-Coto ${ }^{11}$, M. López-Moya9, A. López-Oramas ${ }^{1}$, S. Loporchio ${ }^{22}$, B. Machado de Oliveira Fraga ${ }^{10}$, C. Maggio ${ }^{26}$, P. Majumdar ${ }^{33}$, M. Makariev ${ }^{34}$, M. Mallamaci ${ }^{11}$, G. Maneva ${ }^{34}$, M. Manganaro ${ }^{23}$, K. Mannheim ${ }^{24}$, L. Maraschi ${ }^{3}$, M. Mariotti ${ }^{11}$, M. Martínez ${ }^{5}$, D. Mazin ${ }^{6,15}$, S. Menchiari ${ }^{13}$, S. Mender ${ }^{7}$, S. Mićanović23 ${ }^{23}$ D. Miceli ${ }^{2,49}$, T. Miener ${ }^{9}$, J. M. Miranda ${ }^{13}$, R. Mirzoyan ${ }^{15}$, E. Molina ${ }^{18}$, A. Moralejo ${ }^{5}$, D. Morcuende ${ }^{9}$, V. Moreno ${ }^{26}$, E. Moretti ${ }^{5}$, T. Nakamori ${ }^{35}$, L. Nava ${ }^{3}$, V. Neustroev ${ }^{36}$, C. Nigro ${ }^{5}$, K. Nilsson ${ }^{25}$, K. Nishijima ${ }^{32}$, K. Noda ${ }^{6}$, S. Nozaki ${ }^{31}$, Y. Ohtani ${ }^{6}$, T. Oka ${ }^{31}$, J. Otero-Santos ${ }^{1}$, S. Paiano ${ }^{3}$, M. Palatiello ${ }^{2}$, D. Paneque ${ }^{15}$, R. Paoletti ${ }^{13}$, J. M. Paredes ${ }^{18}$, L. Pavletić ${ }^{23}$, P. Peñil ${ }^{9}$, M. Persic ${ }^{2,50}$, M. Pihet ${ }^{15}$, P. G. Prada Moroni ${ }^{17}$, E. Prandini ${ }^{11}$, C. Priyadarshi ${ }^{5}$, I. Puljak ${ }^{29}$, W. Rhode ${ }^{7}$, M. Ribó ${ }^{18}$, J. Rico ${ }^{5}$, C. Righi ${ }^{3}$, A. Rugliancich ${ }^{17}$, N. Sahakyan ${ }^{28}$, T. Saito ${ }^{6}$, S. Sakurai ${ }^{6}$, K. Satalecka ${ }^{14}$, F. G. Saturni ${ }^{3}$, B. Schleicher ${ }^{24}$, K. Schmidt ${ }^{7}$, T. Schweizer ${ }^{15}$, J. Sitarek ${ }^{12}$, I. Śnidarić Ś $^{37}$, D. Sobczynska ${ }^{12}$, A. Spolon ${ }^{11}$, A. Stamerra ${ }^{3}$, J. Strišković ${ }^{30}$, D. Strom ${ }^{15}$, M. Strzys ${ }^{6}$, Y. Suda ${ }^{27}$, T. Surić ${ }^{37}$, M. Takahashi ${ }^{6}$, R. Takeishi ${ }^{6}$, F. Tavecchio ${ }^{3}$, P. Temnikov ${ }^{34}$, T. Terzić2 ${ }^{23}$, M. Teshima ${ }^{15,6}$, L. Tosti ${ }^{38}$, S. Truzzi ${ }^{13}$, A. Tutone ${ }^{3}$, S. Ubach ${ }^{26}$, J. van Scherpenberg ${ }^{15}$, G. Vanzo ${ }^{1}$, M. Vazquez Acosta ${ }^{1}$, S. Ventura ${ }^{13}$, V. Verguilov ${ }^{34}$, C. F. Vigorito ${ }^{21}$, V. Vitale ${ }^{39}$, I. Vovk ${ }^{6}$, M. Will ${ }^{15}$, C. Wunderlich ${ }^{13}$, T. Yamamoto ${ }^{40}$, and D. Zarić ${ }^{29}$

${ }^{1}$ Instituto de Astrofísica de Canarias and Dpto. de Astrofísica, Universidad de La Laguna, E-38200, La Laguna, Tenerife, Spain. ${ }^{2}$ Università di Udine and INFN Trieste, I-33100 Udine, Italy. ${ }^{3}$ National Institute for Astrophysics (INAF), I-00136 Rome, Italy. ${ }^{4}$ ETH Zürich, CH-8093 Zürich, Switzerland. ${ }^{5}$ Institut de Física d'Altes Energies (IFAE), The Barcelona Institute of Science and Technology (BIST), E-08193 Bellaterra (Barcelona), Spain. ${ }^{6}$ Japanese MAGIC Group: Institute for Cosmic Ray Research (ICRR), The University of Tokyo, Kashiwa, 277-8582 Chiba, Japan. ${ }^{7}$ Technische Universität Dortmund, D-44221 Dortmund, Germany. ${ }^{8}$ Croatian MAGIC Group: University of Zagreb, Faculty of Electrical Engineering and Computing (FER), 10000 Zagreb, Croatia. 9 IPARCOS Institute and EMFTEL Department, Universidad Complutense de Madrid, E-28040 Madrid, Spain. ${ }^{10}$ Centro Brasileiro de Pesquisas Físicas (CBPF), 22290-180 URCA, Rio de Janeiro (RJ), Brazil. ${ }^{11}$ Università di Padova and INFN, I-35131 Padova, Italy. ${ }^{12}$ University of Lodz, Faculty of Physics and Applied Informatics, Department of Astrophysics, 90-236 Lodz, Poland. ${ }^{13}$ Università di Siena and INFN Pisa, I-53100 Siena, Italy. ${ }^{14}$ Deutsches Elektronen-Synchrotron (DESY), D-15738 Zeuthen, Germany. ${ }^{15}$ Max-Planck-Institut für Physik, D-80805 München, Germany. ${ }^{16}$ Instituto de Astrofísica de Andalucía-CSIC, Glorieta de la Astronomía s/n, 18008, Granada, Spain. ${ }^{17}$ Università di Pisa and INFN Pisa, I-56126 Pisa, Italy. ${ }^{18}$ Universitat de Barcelona, ICCUB, IEEC-UB, E-08028 Barcelona, Spain. ${ }^{19}$ Armenian MAGIC Group: A. Alikhanyan National Science Laboratory, 0036 Yerevan, Armenia. ${ }^{20}$ Centro de Investigaciones Energéticas, Medioambientales y Tecnológicas, E-28040 Madrid, Spain. ${ }^{21}$ INFN MAGIC Group: INFN Sezione di Torino and Università degli Studi di Torino, I-10125 Torino, Italy. ${ }^{22}$ INFN MAGIC Group: INFN Sezione di Bari and Dipartimento Interateneo di Fisica dell'Università e del Politecnico di Bari, I70125 Bari, Italy. ${ }^{23}$ Croatian MAGIC Group: University of Rijeka, Department of Physics, 51000 Rijeka, Croatia. ${ }^{24}$ Universität Würzburg, D-97074 Würzburg, Germany. ${ }^{25}$ Finnish MAGIC Group: Finnish Centre for Astronomy with ESO, University of Turku, FI-20014 Turku, Finland. ${ }^{26}$ Departament de Física, and CERES-IEEC, Universitat Autònoma de Barcelona, E-08193 Bellaterra, Spain. ${ }^{27}$ Japanese MAGIC Group: Physics Program, Graduate School of Advanced Science and Engineering, Hiroshima University, 739-8526 Hiroshima, Japan. ${ }^{28}$ Armenian MAGIC Group: ICRANet-Armenia at NAS RA, 0019 Yerevan, Armenia. ${ }^{29}$ Croatian MAGIC Group: University of Split, Faculty of Electrical Engineering, Mechanical 
Engineering and Naval Architecture (FESB), 21000 Split, Croatia. ${ }^{30}$ Croatian MAGIC Group: Josip Juraj Strossmayer University of Osijek, Department of Physics, 31000 Osijek, Croatia. ${ }^{31}$ Japanese MAGIC Group: Department of Physics, Kyoto University, 606-8502 Kyoto, Japan. ${ }^{32}$ Japanese MAGIC Group: Department of Physics, Tokai University, Hiratsuka, 259-1292 Kanagawa, Japan. ${ }^{33}$ Saha Institute of Nuclear Physics, HBNI, 1/AF Bidhannagar, Salt Lake, Sector-1, Kolkata 700064, India. ${ }^{34}$ Inst. for Nucl. Research and Nucl. Energy, Bulgarian Academy of Sciences, BG-1784 Sofia, Bulgaria. ${ }^{35}$ Japanese MAGIC Group: Department of Physics, Yamagata University, Yamagata 990-8560, Japan. ${ }^{36}$ Finnish MAGIC Group: Astronomy Research Unit, University of Oulu, FI-90014 Oulu, Finland. ${ }^{37}$ Croatian MAGIC Group: Ruđer Bošković Institute, 10000 Zagreb, Croatia. ${ }^{38}$ INFN MAGIC Group: INFN Sezione di Perugia, I-06123 Perugia, Italy. ${ }^{39}$ INFN MAGIC Group: INFN Roma Tor Vergata, I-00133 Roma, Italy. ${ }^{40}$ Japanese MAGIC Group: Department of Physics, Konan University, Kobe, Hyogo 658-8501, Japan. ${ }^{41}$ also at International Center for Relativistic Astrophysics (ICRA), Rome, Italy. ${ }^{42}$ now at Department for Physics and Technology, University of Bergen, NO-5020, Norway. ${ }^{43}$ now at University of Innsbruck. ${ }^{44}$ also at Port d'Informació Científica (PIC), E-08193 Bellaterra (Barcelona), Spain. ${ }^{45}$ now at Ruhr-Universität Bochum, Fakultät für Physik und Astronomie, Astronomisches Institut (AIRUB), 44801 Bochum, Germany. ${ }^{46}$ now at Department of Astronomy, University of California Berkeley, Berkeley CA 94720. ${ }^{47}$ also at Dipartimento di Fisica, Università di Trieste, I-34127 Trieste, Italy. ${ }^{49}$ now at Laboratoire d'Annecy de Physique des Particules (LAPP) 